\title{
Observations of electromagnetic radiation of stand EISCAT on Kamchatka
}

\author{
Vladimir Sivokon ${ }^{1}$, Irina Agranat ${ }^{1, *}$ \\ ${ }^{1}$ Institute of Cosmophysical Research and Radio Wave Propagation FEB RAS, ,684034, Kamchatka \\ region, Elizovskiy district, Paratunka, Mirnaya str., 7, Russia
}

\begin{abstract}
In experiments on active influence on an ionosphere powerful short-wave complexes - heater stands are used. As a result of such influence the set of the physical processes [1] which studying represents scientific interest is formed. One of them is formation artificial fieldaligned irregularities. Studying properties natural field-aligned irregularities on Kamchatka [2], authors have come to a conclusion about possibility of realisation of the received approaches in their studying, on short-wave lines of the big extent. In work results of supervision on Kamchatka of displays of active influence on an ionosphere in Tromse (Norway) are resulted.
\end{abstract}

\section{Introduction}

Interest in the fissile impact on an ionosphere does not weaken for a number of reasons, including, applied character. Need of signaling of management on a global scale on pilotless devices is known, for example. Use of the ionospheric wave duct possessing minimum losses on long-distance trasses can be one of solutions of this problem. But in the presence at the similar channel of a number of advantages it is necessary to resolve an issue of its efficient exaltation. One of ways of "powering" of the channel is scattering on the simulated small-scale inhomogeneities of an ionosphere which are formed under the influence of a potent heating wave. In this case the effectiveness of exaltation is defined by type of scattering and its spatial configuration. As a rule, it is considered that aspect scattering when falling an electromagnetic wave on the magnetofocused inhomogeneities of an electron concentration scattering happens in a cone which top is in the place of their arrangement takes place. But if to consider set of the magnetofocused radiators as the system of reradiators it is possible to draw a conclusion that the effectiveness of exaltation of the ionospheric channel depends on their dimensional orientation and situation in it.

For studying of topology of these inhomogeneities there are various methods, including, methods of a trial wave [3] and aspect scattering [4]. In case of the former the modified area of an ionosphere is periodically irradiated with auxiliary radiation and on change of its characteristics assessment of variations of its properties is made. In a method of aspect scattering the auxiliary transmitter remote from the venue on rather big removal is used, and the dispelled signal is accepted in point located so that trajectories of a direct signal and

Corresponding author: agranat@ikirru 
disseminated on the modified area made a corner, the close to 90 degrees. It allows to carry out space selection of direct and dispelled signals and as radiation happens continuously, to estimate speed occurring in the modified area of movements of plasma on the doppler shift of frequency. As the auxiliary transmitter the broadcasting station is, as a rule, used. This approach is inapplicable to studying of the magnetofocused inhomogeneities as polarizable coordination - polarization of a wave at such stations horizontal is not carried out.

As the magnetofocused inhomogeneities are extended along power lines of a magnetic field of Earth, their dimensional orientation is defined by properties of a magnetic field in the venue of experiments. For Tromse, according to the Kyoto university, the magnetic dip is equal 78.230, and the magnetic declination makes 8.740. Therefore, inhomogeneities will be focused at an angle 78.23 degrees in the vertical plane. Follows from the same data that in the horizontal plane the true direction on a geographical pole will differ by 8.74 degrees. We will return to these parameters a bit later, and now we will consider separate nuances of the fissile impact on an ionosphere.

For increase in effectiveness of impact on an ionosphere there is a number of technologies. Let's remind those from them which are essential to our observations: the selection polarizable exaltation of characteristic waves and effect of a magnetic zenith.

When falling a wave of the arbitrariest polarization on an ionosphere there is a formation of two types of waves: ordinary and unusual [5]. Waves differ in polarizable characteristics and if on the transferring party to create the electromagnetic field coinciding in this parameter with one of waves, then this wave will be mainly excited. Ordinary and unusual waves have different refractivities and extend in an ionosphere with different absorption indexes. Respectively extent of indignation of plasma and amount of the formed ionospheric inhomogeneities will be waves of various polarization, various at radiation. Therefore, information on type of the polarization applied in a heating experiment is important for our observations.

The effect of a magnetic zenith consists in essential increase in effectiveness of influence at the radiation of electromagnetic waves not upright up, and along power lines of a magnetic field of Earth. Therefore, proceeding from parameters of a magnetic field of Earth, the maximum of a directional diagram of the radiating antenna will be focused at an angle 78.23 degrees in the vertical plane, and at an angle 188,74 degrees in the horizontal plane.

\section{Method of investigations}

Let's estimate probability of direct reception of a heating wave on TromsePetropavlovsk-Kamchatsky Route. For this purpose we use the VOACAP program, including its on-line version and technical parameters of transmitting antennas of the website EISCAT [8]. The VOACAP program estimates distance between Tromse and Petropavlovsk-Kamchatsky at 6021 kilometer, the azimuth at the same time makes 24,6 degrees. The difference between a principal direction of radiation of a transmitting antenna and an azimuth will make 188,74-24,6 =164,14 degrees. Directional properties of transmitting antennas depend on quantity of elements in a lattice and the used frequency, the figure 1.

According to data, the presented head of experiments Blagoveshchenskaya N.F., in experiments used an antenna lattice number 2. Follows from the schedule that directional diagram width in the vertical plane on the level of $3 \mathrm{~dB}$ will be from 15 to 10 degrees depending on frequency. In experiments the frequency of $5421 \mathrm{kHz}$ for which width of a directional diagram is about 10 degrees, fig. 1 was used. Therefore, emitted energy is generally concentrated within 73-83 degrees in the vertical plane. Meanwhile, calculations with use of VOACAP show, fig. 2 that for reception of a heating wave on Kamchatka 
radiation has to occur within 3-10 degrees in the vertical plane.

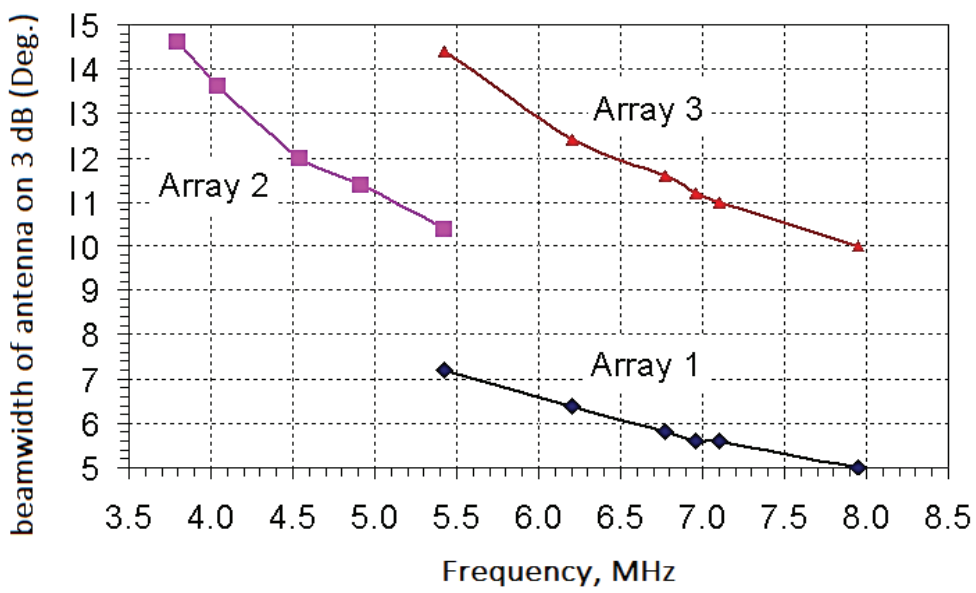

Fig. 1. Directional properties of transmitting antennas of the heating EISCAT stand.

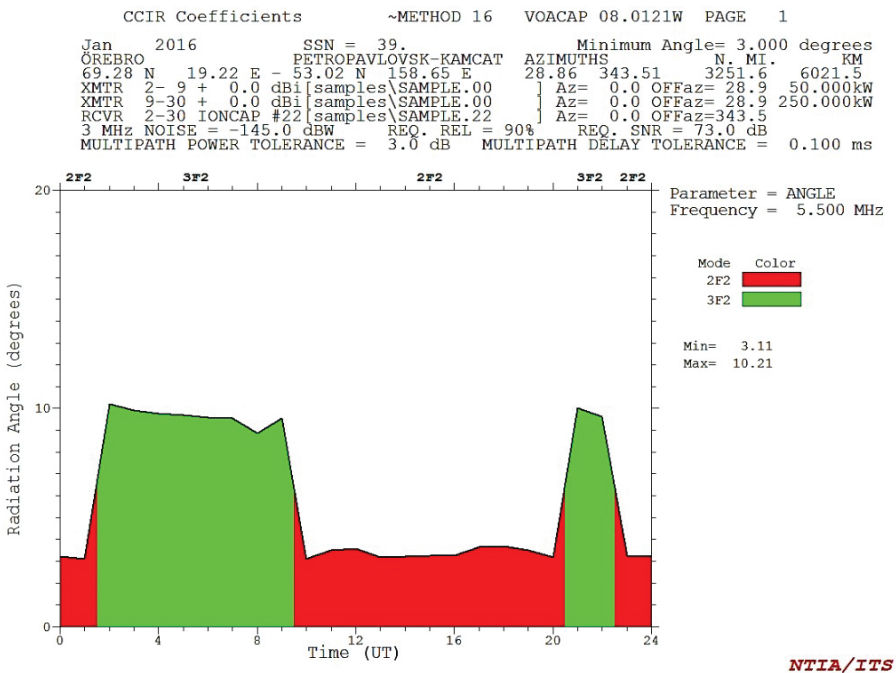

Fig. 2. Estimated values of a corner of radiation in the vertical plane for Tromse-PetropavlovskKamchatsky Route.

From the carried-out analysis it is possible to draw a conclusion that reception of the heating wave extending in a wave guide ionosphere-Earth is improbable.

For definition of conditions of distribution in an interlayered wave guide we use the most probable model in which distribution happens in channel between layers of $\mathrm{E}$ and $\mathrm{F}$. Information on a possibility of existence of such condition can be obtained from ionograms of the place of influence, fig. 3 


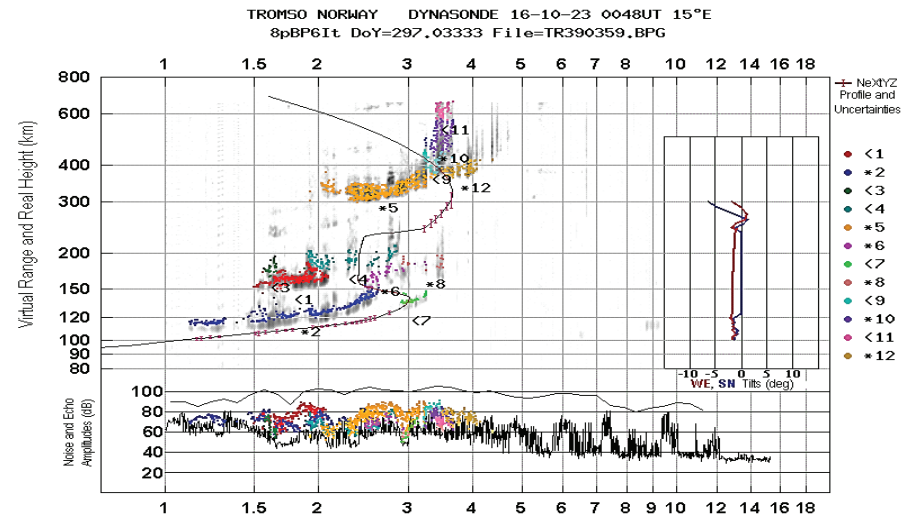

Fig. 3. Ionogram to Tromsø on October 23, 201600.48 UT.

In fig. 3 reflections at the level of a layer of $\mathrm{E}$ and $\mathrm{F}$ are visible that can demonstrate existence of conditions for formation of an interlayered ionospheric wave guide in the venue of impact on an ionosphere.

The majority of the radio route of Tromse-Petropavlovsk-Kamchatsky passes in polar and subpolar latitudes (fig. 4) of which the serious violations of a radio communication caused by magnetic storms are characteristic.

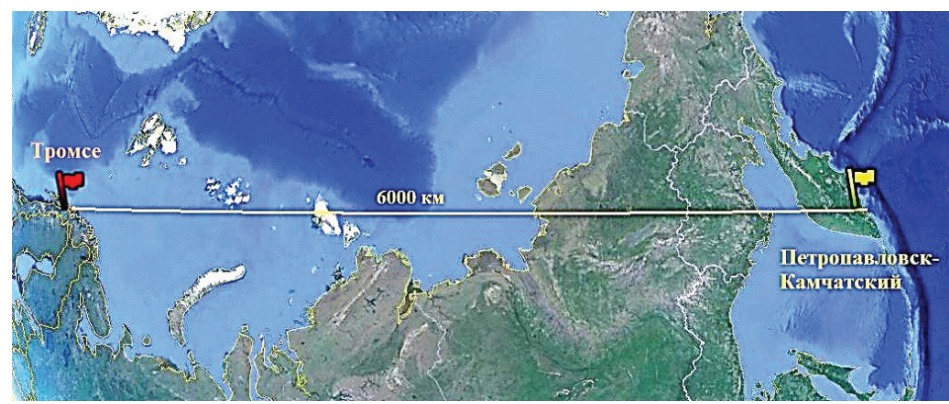

Fig. 4. Radio route of Tromse-Petropavlovsk-Kamchatsky.

Besides in the conditions of the indignant magnetic field of Earth in polar regions the moving ionospheric agitations which can significantly affect results of observations are formed. Using data of the university of Kyoto, Japan, we will estimate a condition of a magnetic field of Earth during experiments. As the parameter in which assessment is made we use Dst variations as unlike the planetary magnetic index $\mathrm{Kr}$, Dst allows to estimate not only extent of indignation of a magnetic field, but also an indignation phase. Earlier it was shown that at the selection polarizable exaltation of characteristic waves in an ionosphere the phase of indignation of a magnetic field plays an important role [6]. As a result of processing of magnetic data it was received distribution on fig. 5. 


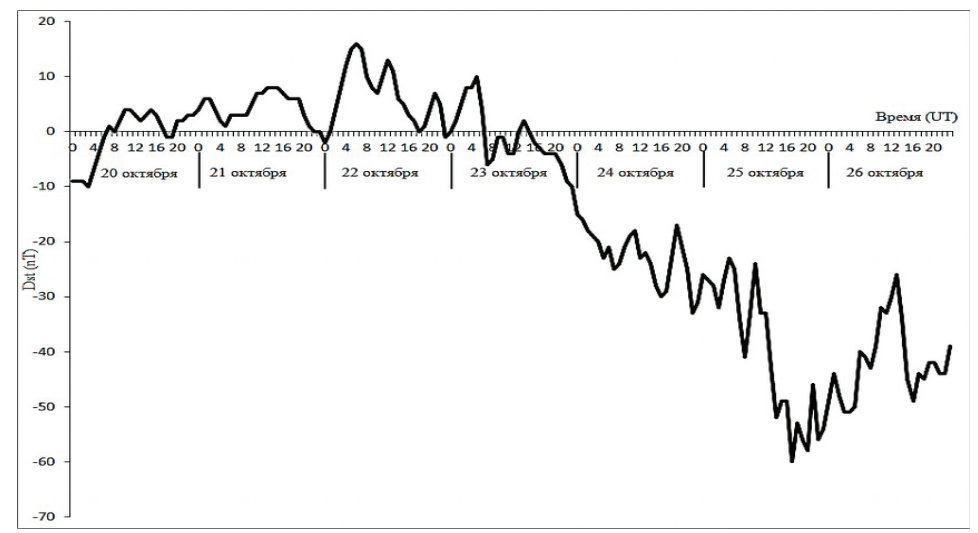

Fig. 5. Variations of a magnetic field of Earth during the period from October 20 to October 26, 2016.

Experiments were made after 12 clocks of world time, and for this time slice, as appears from fig. 5, for reception and the analysis of heating radiation are optimum on October 2023, 2016.

We will give spectrograms of radiations as an example of the made observations at a frequency of $5423 \mathrm{kHz}$ on October 21 at 14 hours 13 minutes, fig. 6 and 23 of October at 12 hours 43 minutes fig. 7

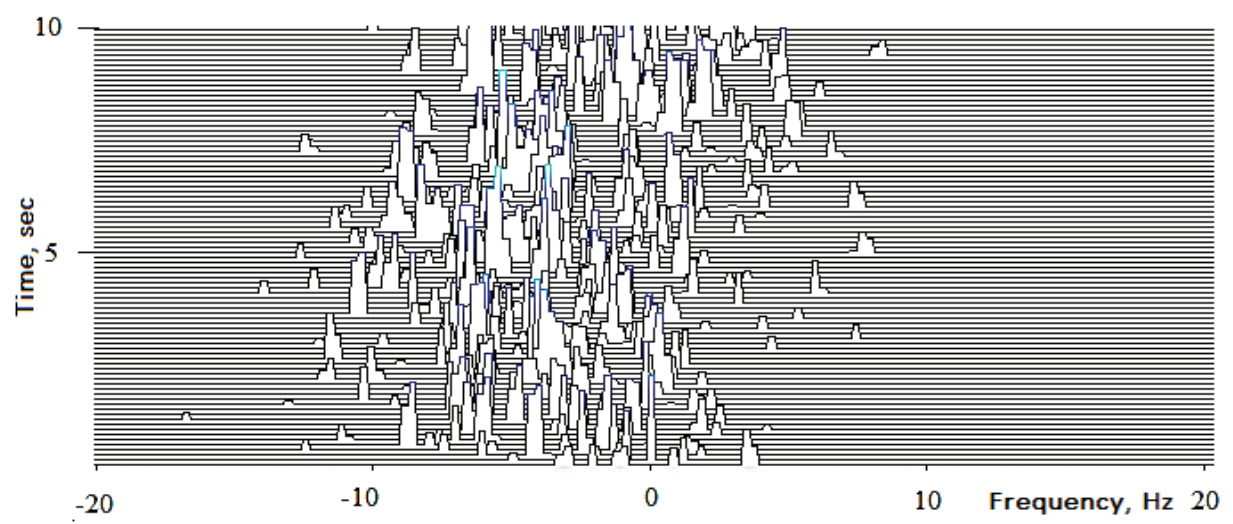

Fig. 6. Spectrogram of radiation on October 21, 2016. 


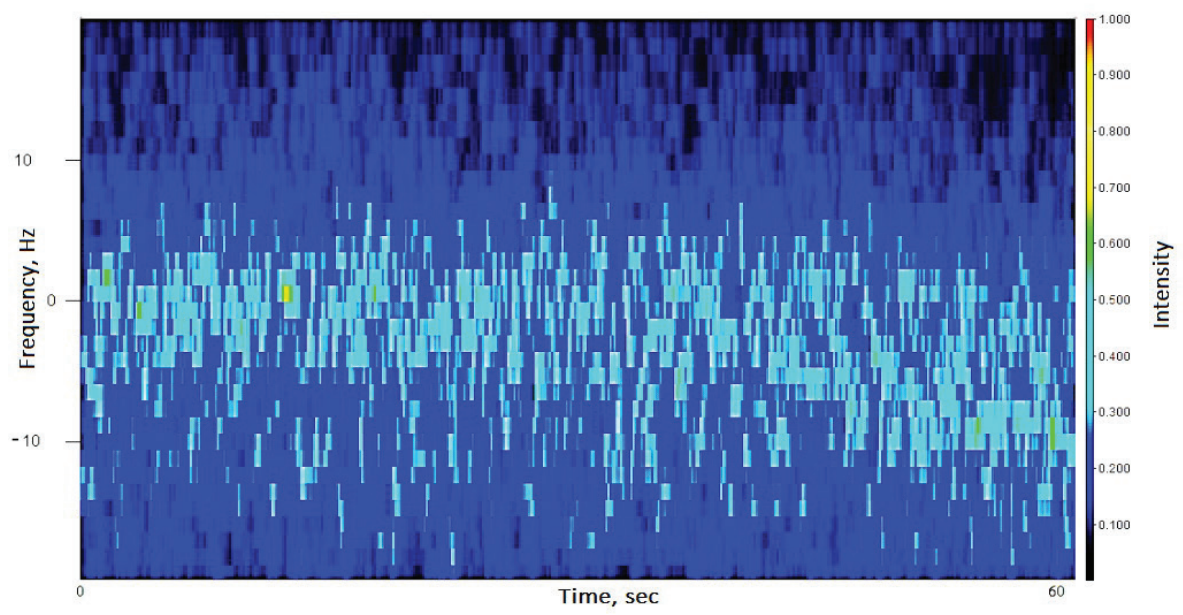

Fig. 7. Spectrogram of radiation on October 23, 2016.

For descriptive reasons ranges of radiations are presented in different formats, and it is apparent that in both cases the broadening of a range and its variation in time, mainly towards the negative detuning of frequency takes place.

Follows from data of the website EISCAT that stability of frequency of the master oscillator used in experiments makes the Hz. Stability of frequency of the radio-receiving device makes the Hz. Then, if to proceed from parameters of the least stable device - the radio-receiving - the absolute instability of frequency caused by technical means will be $0,05423 \mathrm{~Hz}$ that is tens times less than the detuning recorded in an experiment.

The shift on frequency can be caused by existence of the moving ionospheric agitations which main mechanism of emergence are geophysical indignations in auroral area. As for the analysis the data obtained in instants when the magnetic field of Earth was quiet are used it is possible to consider that the probability of manifestation of the moving ionospheric agitations in a frequency shift is improbable.

\section{Results of investigation and discussion}

When carrying out experiments with use for diagnostics of a method of aspect scattering $[4,7]$ frequency shifts of the diagnostic transmitter of the same order, as well as recorded by us were received. In general, they correspond to the data obtained by us in these observations and in work [2]. But, as, it is considered that these indignations consist of the magnetofocused inhomogeneities [8], it is necessary to specify effectiveness of scattering on them. For this purpose we will use the drawing provided in work [8], fig. 8. 


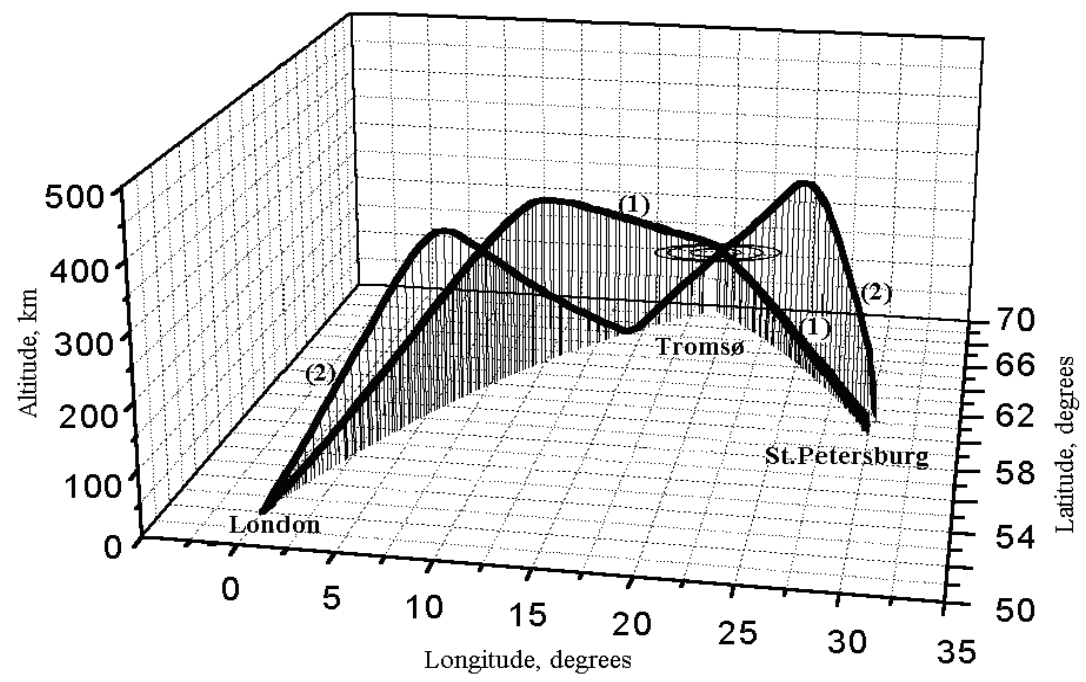

Fig. 8. Geometry of aspect scattering.

Let's remind that the transmitting antenna in London has horizontal polarization. The magnetofocused inhomogeneities over Tromse are located at an angle 78 degrees. In the figure 8 two fashion 1 and 2 are shown, each of which has refraction in an ionosphere, i.e. when falling on area of influence, the wave will be elliptically polarized. Therefore, unlike observations on Kamchatka [2], in experiments [9] it is not possible to provide completely polarizable coordination with the magnetofocused inhomogeneities. As the frequency of a heating wave and a frequency shift is known, it is possible to determine the speed of inhomogeneities which sizes for thirty second intervals of the experiments made on October 21 at 14 hours 13 minutes, are shown in fig. 9.

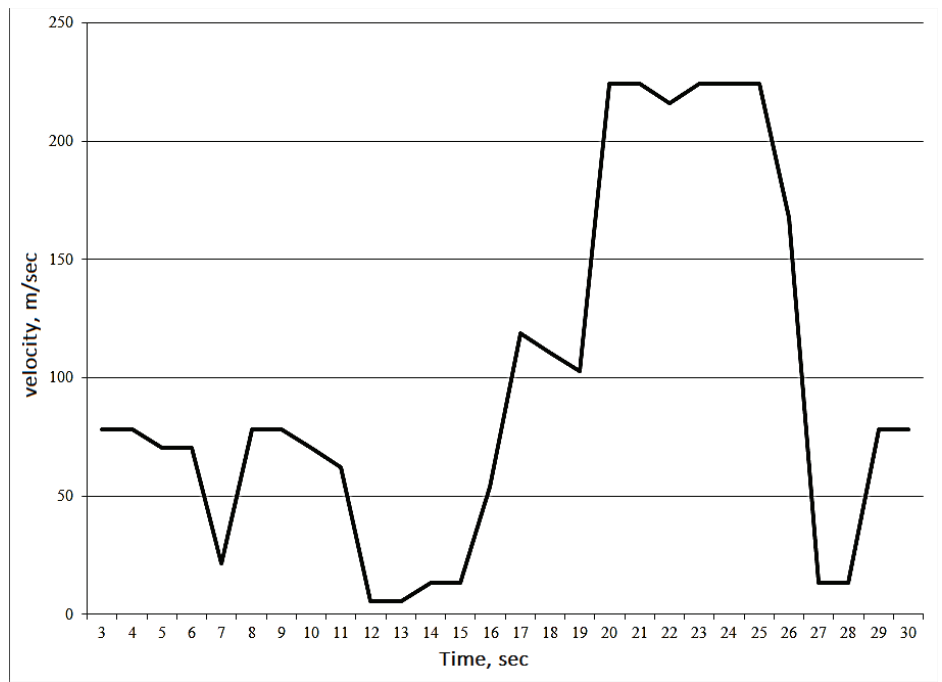

Fig. 9. Speed of the magnetofocused inhomogeneities 


\section{Conclusions}

The possibility of distant selection diagnostics of properties of the magnetofocused inhomogeneities without use of auxiliary transmitters is shown. Observations showed existence in the radiation of the frequencies shift which size is comparable to the sizes received with use of a method of aspect scattering. Peculiar properties Route distribution of a signal allows to allocate as the most probable interlayered ionospheric waveguide. The specifics of exaltation of an interlayered ionospheric wave guide allow to assume that the frequencies shift is caused by driving of the magnetofocused inhomogeneities.

\section{References}

1. Gurevich A.V. Uspehi fizicheskih nauk, 177, 11, 1145-1177 p. (2007)

2. Sivokon V.P., Kalugin I.A., Kobylkin V.S., Popov A.V. Vestnik KamchatGTU, 37, 14$18 \mathrm{p}$.

3. Grach S.M., Komrakov G.P., Schvartz M.M., Yurishev M.A. Izvestiya vyschih uchebnyh zavedeniy. Radiofizika, 41, 8, 966-977 p

4. Blagoveshchenskaya N. F., Borisova T. D., Kornienko V. A., Robinzon T.R., Yoman T.K., Frolov V.L., Ritveld M.T. Solnechno-zemnaya fizika, 2, 12, 206-209 p. (2008)

5. K.Devis. Radiovolny v ionosphere, 504 p. (1973)

6. Sivokon V.P., Druzhin G.I. Geomagnetism i aeronomiya, 46, 4, 521-524 p. (2006)

7. Blagoveshchenskaya N. F., Borisova T. D., Kornienko V. A., Moskvin I. V., Rietveld M. T., Frolov V. L., Uryadov V. P., Kagan L. M., Yampolski Yu. M., Galushko V. L., Koloskov A. V., Kasheev S. B., Zalizovski A. V., Vertogradov G. G., Vertogradov V. G., Kelley M. C. 24, 2333-2345 p. (2006)

8. Borisova T. D., Blagoveshchenskaya N. F., Moskvin I. V., Rietveld M. T., Kosch M. J., Thid'e B. Geophys., 20, 1479-1486 p. (2002) 